\title{
Sachgerechte Finanzierung der Sozialversicherung als politische Aufgabe
}

\begin{abstract}
Wie die Sozialversicherungen durch Beiträge und Steuern dem Aufgabenzweck angemessen finanziert werden können, gehört nach wie vor auf die politische Tagesordnung. Zwar ist manches - zum Teil aber Widersprüchliches - entschieden worden. Trotzdem bleibt viel zu tun. Auf die Gründe dafür und wie dies realisiert werden kann, wird nachfolgend eingegangen. Dabei sind allerdings Unterschiede zwischen den Sozialversicherungszweigen ebenso zu beachten wie die jeweils für sie maßgebenden Konzeptionen. Am Beispiel der gesetzlichen Rentenversicherung wird schließlich gezeigt, wie eine veränderte Finanzierung zentrale Bedeutung für den Erhalt eines angemessenen Leistungsniveaus besitzt.
\end{abstract}

\section{1 \\ Einleitung}

In diesem Beitrag geht es vornehmlich um die richtige Finanzierungsart, ${ }^{1}$ mit der der Finanzbedarf der Sozialversicherung gedeckt werden kann - durch Beiträge (mit denen eine Gegenleistung erworben wird) oder durch Steuern (mit deren Zahlung kein Anspruch auf eine Gegenleistung verbunden ist). ${ }^{2}$ Für Beiträge wie auch für Steuern gibt es viele Gestaltungsmöglichkeiten hinsichtlich Personenkreis, Bemessungsgrundlage und Tarif.

Die Beitragsfinanzierung steht seit einiger Zeit auch deshalb in der Kritik, weil eine Erosion der Beitragsbasis zu verzeichnen ist. Dafür gibt es verschiedene Gründe, von denen vier erwähnt seien:

(1) Der Anteil der beitragspflichtigen Bruttolohnsumme am Wirtschaftsprodukt nimmt ab.

(2) Die Zahl der beitragspflichtigen Erwerbstätigen an allen Erwerbstätigen geht beispielsweise durch geringfügige und selbstständige Erwerbstätigkeit zurück.

(3) Der Gesetzgeber reduziert die beitragspflichtigen Bruttoentgelte, beispielsweise durch die beitragsbefreite (Brutto-) Entgeltumwandlung (Schmähl/Oelschläger 2007).

(4) Die soziale Sicherung wird auf Träger privater Vorsorge verlagert, was die Verpflichtung oder Notwendigkeit mit sich bringt, Beiträge an private Versicherungen und nicht an Sozialversicherungsträger zu entrichten.
Sozialversicherungen stellen Mischsysteme aus zwei grundlegend verschiedenen Systemtypen dar: einem Versicherungssystem, bei dem eine enge Beziehung zwischen Vorleistung (dem Beitrag) und Gegenleistung besteht, und einem Steuertransfersystem, bei dem die Leistungen in der Regel ohne Bezug zu früheren monetären Vorleistungen erfolgen (Schmähl 2009a, Kap. 3). Typisch für (reine) Steuertransfersysteme sind (bedürftigkeitsgeprüfte) Fürsorgesysteme wie auch Versorgungssysteme. Allerdings kann in Sozialversicherungen das Mischungsverhältnis zwischen diesen beiden Systemtypen unterschiedlich sein. Es kann sich im Zeitablauf ändern und zwischen verschiedenen Sozialversicherungszweigen unterscheiden: So existieren in Deutschland erhebliche Unterschiede darin, in welchem Verhältnis die jeweiligen monetären (Vor-)Leistungen - also Beitragszahlungen - zu den Gegenleistungen stehen. Eine enge Beziehung zwischen Höhe des Beitrags und Höhe der Gegenleistung ist dort am besten zu realisieren, wo Geldleistungen dominieren, wie in der Gesetzlichen Rentenversicherung (GRV). Anders ist es, wenn primär Sachleistungen gewährt werden, wie insbesondere in der Gesetzlichen Krankenversicherung (GKV): Dort ist die Leistungshöhe im Prinzip unabhängig von der Höhe der Beiträge bzw. vom laufenden oder früheren Einkommen; maßgebend ist der medizinisch festgestellte und durch leistungsrechtliche Regelungen begrenzte Bedarf. ${ }^{3}$

Zunächst werden folgende Fragen erörtert:

Wann ist in Sozialversicherungssystemen Beitrags- oder aber Steuerfinanzierung angemessen? Welche Probleme ergeben sich, wenn an die Stelle einer vom Aufgabenzweck her erforderlichen Steuer- finanzierung die Finanzierung durch Beitragseinnahmen tritt? Welche Argumente sprechen gegen eine derartige „Fehlfinanzierung", und wie kann diese beseitigt werden?

Anschließend werden Vorschläge $\mathrm{zu}$ Änderungen der Ausgestaltung von Beitragsfinanzierung kurz diskutiert, und zwar die Ausweitung der Bemessungsgrundlage für Versicherte sowie für Arbeitgeber bzw. Unternehmen („Wertschöpfungsabgabe“). Schließlich wird für die GRV die Frage aufgeworfen, welche zentrale Rolle eine aufgabenadäquate Finanzierung in einer Strategie zur weiteren Entwicklung der GRV spielt, wenn diese als

\footnotetext{
1 Kritische Anmerkungen zur vorherrschenden Meinung der angeblich zweifelsfreien Überlegenheit des Finanzierungsverfahrens Kapitalfundierung in der Alterssicherungspolitik bietet Schmähl (2009a, S. 31-34 sowie die Kapitel 10-13)

2 Es gibt allerdings immer wieder im In- wie Ausland Bestrebungen, den Unterschied zwischen Beitrag und Steuer zu verwischen. Die Weltbank propagiert beispielsweise für Tschechien einen integrierten Abgabeneinzug von Beiträgen und Steuern, da beide Abgaben faktisch Steuern seien, was auch manchmal in der deutschen Diskussion zu vernehmen ist (Ruland 2009).

3 Im Hinblick auf die Beziehung zwischen Vor- und Gegenleistung kann zwar auch bei Sachleistungen davon gesprochen werden, dass hier ein Anspruch aufgrund der Vorleistung besteht, allerdings (nur) dem Grunde nach, während die Höhe des Anspruchs (also der Gegenleistung) von anderen Faktoren abhängig ist.
}

Winfried Schmähl, Dr., Professor im Ruhestand an der Universität Bremen. Arbeitsschwerpunkte: Ökonomische Analyse der Sozialpolitik, Einkommensverteilung und -umverteilung. e-mail:w.schmaeh/@t-online.de 
beitrags- und lohnbezogene Einrichtung mit Lohnersatzcharakter auch für die $\mathrm{Zu}$ kunft erhalten bleiben soll.

\section{Aufgabenadäquate Finanzierung}

\subsection{BEGRÜNDUNGEN}

Welches die geeignete Finanzierungsart für Ausgaben der Sozialversicherung ist, hängt von den damit verbundenen bzw. erwarteten Wirkungen ab und sollte vor allem im Hinblick auf Konzeption und Ziele des jeweiligen sozialen Sicherungssystems entschieden werden.

Während in der GRV bereits seit der Kaiserzeit ein gewichtiges steuerfinanziertes Element besteht, spielte die Steuerfinanzierung in der GKV bis vor Kurzem kaum eine Rolle. Erst in jüngster Zeit kam die Steuerfinanzierung in der GKV auf die politische Tagesordnung, um der Steigerung der Lohnnebenkosten entgegenzuwirken und um aus verteilungspolitischen Gründen die Lasten zwischen Privathaushalten zu verändern.

Dies sind auch die beiden zentralen Argumentationsstränge, wenn darüber diskutiert wird, wie die Mittel für die Sozialversicherung aufgebracht werden können. Will man beurteilen, welches Finanzierungsinstrument der Aufgabe adäquat ist, so sind Kriterien erforderlich: Aus dem Vergleich von dem, was sein sollte, und dem, was tatsächlich ist, ergibt sich, ob und in welchem Umfang eine „Fehlfinanzierung" besteht. Darunter wird hier verstanden, dass es in der Sozialversicherung Aufgaben und Ausgaben gibt, die in der Realität zwar aus Beiträgen finanziert werden, die gemäß ihrem Aufgabenzweck jedoch aus Steuern zu finanzieren wären, wofür häufig der missverständliche Begriff der „versicherungsfremden Leistungen“"verwendet wird.

Ausgangspunkt der weiteren Überlegungen ist folgende Leitidee: Wird in der Sozialversicherung ${ }^{4}$ gezielt interpersonelle Einkommensumverteilung angestrebt, soll dies aus dem Steueraufkommen finanziert werden. Das bedeutet: Wenn durch die Gestaltung der Versicherungsbedingungen gezielt Personengruppen bessergestellt bzw. andere stärker belastet werden, wenn also allgemeine Staatsaufgaben über die Sozial- versicherung abgewickelt werden (wie z. B. familienpolitische Maßnahmen oder bedürftigkeitsgeprüfte Leistungen), so sollten die damit verbundenen Ausgaben weitgehend aus allgemeinen Haushaltsmitteln der Gebietskörperschaften (hier vereinfachend: aus dem Steueraufkommen), nicht aber aus (vorwiegend) am Arbeitsentgelt oder an Lohnersatzleistungen ${ }^{5}$ anknüpfenden Beiträgen finanziert werden. Selbst die Bundesbank, die lange Zeit eher den Blick auf den (zu reduzierenden) Umfang der Sozialausgaben richtete, befürwortet neuerdings hinsichtlich der Finanzierung von Sozialversicherungsleistungen „eine ordnungspolitisch geleitete Herangehensweise $[\ldots]$. Demnach wären Steuerzuschüsse vom Prinzip her am Umfang der versicherungsfremden Leistungen $\mathrm{zu}$ bemessen beziehungsweise allgemeine Umverteilungsaufgaben über Steuern zu finanzieren" (Deutsche Bundesbank 2008, S. 54). Denn wenn Ausgaben für Umverteilungszwecke aus lohnbezogenen Beiträgen finanziert werden, so resultieren daraus negativ zu bewertende ökonomische Folgen, von denen hier drei genannt seien:

(1) Verteilungspolitisch negativ zu bewerten ist dies, da im Falle der Beitragsfinanzierung - im Unterschied zur Steuerfinanzierung - nur der begrenzte Kreis der Versicherungspflichtigen zur Finanzierung der Umverteilungsaufgaben herangezogen wird, sich aber nicht alle Steuerpflichtigen bzw. Steuerzahler an der Finanzierung beteiligen. Die Beitragszahlung bezieht sich im Falle der versicherungspflichtigen Beschäftigten in Deutschland ausschließlich auf das Bruttoarbeitsentgelt, und zwar nur bis zur jeweiligen Beitragsbemessungsgrenze. Dies widerspricht den üblicherweise vertretenen Vorstellungen über die fiskalische Leistungsfähigkeit als Kriterium für die Lastverteilung bei der Finanzierung allgemeiner Staatsaufgaben.

(2) Außerdem erhöhen sich infolge der Arbeitgeberbeiträge (ceteris paribus) die Lohnkosten für solche Aufgabenzwecke, die nichts mit der monetär bewerteten Leistung des Faktors Arbeit zu tun haben. Damit geht tendenziell ein Anreiz zur Substitution von Arbeit durch Kapital einher, da das Faktorpreisverhältnis zulasten des Faktors Arbeit verändert wird. Dieses allokative Argument betrifft insbesondere die Arbeitsnachfrage, die u. a. von den gesamten
Lohnkosten abhängt, zu denen die Arbeitgeberbeitragszahlungen gehören. ${ }^{6}$

Durch hohe und „überhöhte“ Arbeitgeberbeiträge werde - so ein in der politischen Diskussion gewichtiges Argument die Wettbewerbsposition von Unternehmen (international wie auch gegenüber Schattenaktivitäten) verschlechtert, zumal wenn die Überwälzung der (Lohn-)Kosten nur schwer oder mit erheblicher Zeitverzögerung möglich ist. Aufgrund der Intensivierung des internationalen Wettbewerbs seien die Möglichkeiten zur Abwälzung von Kosten in Preise begrenzt. Insbesondere in Zeiten, in denen das Arbeitsangebot die Arbeitsnachfrage (deutlich) übersteigt, bestehe die Gefahr, dass hieraus zusätzlich verschlechterte Beschäftigungsbedingungen resultieren. Überhöhte Sozialbeiträge als Folge der Fehlfinanzierung wirken dann beschäftigungspolitisch negativ. Anders formuliert: Wird die Fehlfinanzierung abgebaut und werden damit einhergehend die Sozialbeiträge gesenkt, würde dies einen positiven Einfluss auf die Beschäftigungssituation ausüben. Die Forderung nach einer Senkung der Lohnnebenkosten bzw. einer Senkung der Arbeitgeberbeiträge, wird seit Langem erhoben und wurde z. B. im letzten Jahrzehnt neben dem Verweis auf demografische Veränderungen zur zentralen Begründung für inzwischen eingeleitete Reformmaßnahmen insbesondere in der GRV. Auch wenn politisch immer wieder eine Senkung der Beiträge als Ziel proklamiert wurde, so entlastete sich bis in die jüngste Zeit der Bundeshaushalt dennoch immer wieder zulasten der Sozialversicherungen und erhöhte damit die Beitragsbelastung. Diese erfolgte auch durch die politische Entscheidung zur zeitlich unbegrenzten Fortführung der beitragsbefreiten Entgeltumwandlung oder durch die Reduzierung der Beitragszahlungen des Bundes für Bezieher von Arbeitslosengeld II an GKV und GRV.

$4 \quad$ Nachfolgend bleiben Sondersysteme - wie die für Landwirte oder Bergleute - ausgeklammert. Dort spielen strukturpolitische Ziele eine wichtige Rolle und führen (so in Deutschland) zu einem hohen Anteil der Steuerfinanzierung.

5 Wie z.B. Beiträge zur GKV von gesetzlichen und betrieblichen Renten.

6 Das Argument, lohnbezogene Sozialbeiträge seien allein eine Steuer auf Arbeit und würden damit die Arbeitsnachfrage schwächen, kann allerdings nicht einfach auf solche Systeme bezogen werden, bei denen mit der Höhe des Beitrags auch die Höhe des Anspruchs auf Gegenleistung verbunden ist, wie in der GRV. 
In der deutschen wirtschaftspolitischen Diskussion der vergangenen Jahre wurde allerdings die Bedeutung der Arbeitgeberbeiträge für die Lohnkosten zumeist erheblich überbetont, um damit politisch Druck in Richtung auf die Reduzierung von Sozialausgaben auszuüben (Schmähl 2009b).

(3) Werden allgemeine Staatsaufgaben aus Beiträgen finanziert, so wird die LeistungsGegenleistungs-Beziehung verwässert, also die Beziehung zwischen Vorsorgebeitrag (bzw. dem Einkommen, das der Beitragszahlung zugrunde liegt) und den späteren Versicherungsleistungen. Dies ist besonders dort relevant, wo die Höhe der Geldleistung maßgeblich von der Höhe der Beitragszahlung abhängt. Solche „überhöhten" Beiträge können sich damit nicht nur negativ auf die Arbeitsnachfrage, sondern auch negativ auf das Arbeitsangebot auswirken, wenn sich aus Sicht der Arbeitnehmer die Beitragszahlung für sie wenig oder sogar zunehmend weniger „lohnt“, da der Beitrag infolge der Fehlfinanzierung mehr und mehr den Charakter einer Steuer erhält. Denn wie die Arbeitnehmer die Minderung ihres Nettoentgelts infolge ihrer Beitragszahlungen beurteilen und wie sie darauf gegebenenfalls reagieren, dürfte mit davon abhängen, inwieweit sie erwarten, dass ihren Beiträgen auch eine als adäquat anzusehende Gegenleistung gegenübersteht. Je deutlicher für die Versicherten wird, dass mit ihrer Beitragszahlung auch ein Anspruch auf eine als angemessen beurteilte Gegenleistung erworben wird, umso eher dürften die Versicherten den Beitrag als einen Preis für den Erwerb sozialer Sicherheit durch Versicherungsleistungen ansehen (Deutsche Bundesbank 2008, S. 5) und daher anders bewerten als eine Steuerzahlung. Infolgedessen werden sie weniger Versuche zur Vermeidung unternehmen. Auch bei Tarifverhandlungen spielt dieser Gesichtspunkt eine Rolle, indem mehr über den Brutto- als den Nettolohn verhandelt wird, wenn der Sozialversicherungsbeitrag überwiegend Preischarakter besitzt.

\subsection{AUSMAß DER "FEHLFINANZIERUNG"}

Trotz dieser negativen Effekte ist das Ausmaß der „Fehlfinanzierung“ in der deutschen Sozialversicherung in allen Zweigen beträchtlich, selbst wenn in manchen Fällen über die konkrete Abgrenzung der aus
Steuern zu finanzierenden Ausgaben und damit über das Ausmaß der „Fehlfinanzierung" unterschiedliche Vorstellungen bestehen.

Aus dem Vergleich der „versicherungsfremden Leistungen“, die aus Steuermitteln $\mathrm{zu}$ finanzieren wären, mit den Steuermitteln, die den Sozialversicherungen tatsächlich zufließen, ergibt sich das Ausmaß der Fehlfinanzierung und damit zugleich das Potenzial zur Senkung von Beitragssätzen. Dies kann bei einer Gesamtsumme der Beitragssätze von über $40 \%$ (wie im Herbst 2008) mit 7 bis 9 Prozentpunkten beziffert werden (Schmähl 2007a).

Dabei ist zu berücksichtigen, dass (bei versicherungspflichtiger Beschäftigung) zusätzlich zu den paritätisch von Arbeitnehmern und Arbeitgebern gezahlten Beiträgen auch allein von Versicherten zu zahlende (Zusatz-)Beiträge erhoben werden. So erfolgte eine Verschiebung des bislang hälftigen Aufteilungsverhältnisses zwischen Arbeitnehmern und Arbeitgebern z. B. in der GKV durch einen seit 2005 allein von den Versicherten (Arbeitnehmern wie Rentnern) zu zahlenden Zusatzbeitrag von $0,9 \%$. Dieser bleibt auch nach Einführung des Gesundheitsfonds im Jahre 2009 bestehen, mit dem die Festlegung eines einheitlichen Beitragssatzes für alle gesetzlichen Krankenkassen erfolgt. Erwartet wird, dass in einzelnen Kassen schon bald weitere Zusatzbeiträge erforderlich werden, die wiederum allein von den Versicherten zu tragen sind. Ausnahmen von der paritätischen Finanzierung gibt es auch in der Pflegeversicherung, da bei ihrer Einführung für die Arbeitgeber außer in Sachsen eine Kompensation erfolgte und zudem Rentner inzwischen den vollen Beitrag zahlen. Außerdem existiert hier ein Zusatzbeitrag von 0,25 \% für „Kinderlose“.

Das Verhältnis der Zahllast von Vorsorgeaufwendungen zwischen Arbeitgebern und Versicherten verschiebt sich auch dann, wenn das Leistungsniveau der Sozialversicherung generell reduziert wird wie in der GRV - und die Versicherten auf private bzw. betriebliche Systeme verwiesen werden, um das bisherige Leistungsniveau aufrechtzuerhalten oder wenn - wie in der GKV - Zuzahlungen der Kranken erforderlich sind.

Die quantitativ größten Posten der Fehlfinanzierung stellen familienpolitische Leistungen in der GKV dar (mit 2,5 bis 3 Beitragspunkten $)^{7}$ sowie die nur nach Einkommensüberprüfung gezahlten Hinter- bliebenenrenten in der GRV (mit etwa 3,5 Beitragspunkten).

Nach langjährigen erheblichen Widerständen gegen eine Steuerfinanzierung familienpolitisch motivierter Ausgaben in der GKV soll nun 2009 ein Steuerzuschuss von $7 \mathrm{Mrd}$ €, ab 2010 von 11,5 Mrd. € bis schließlich auf $14 \mathrm{Mrd}$. $€$ steigend gezahlt werden. Diese Beträge werden allerdings nur einen Teil der Fehlfinanzierung in der GKV decken, die mit gut $30 \mathrm{Mrd}$ € oder etwa $20 \%$ des gesamten Ausgabenvolumens von gut $155 \mathrm{Mrd}$. $€$ anzusetzen ist. Würden an die Stelle der „überhöhten“ Beitragszahlungen tatsächlich in ausreichendem Umfang Steuermittel treten, dann lägen die Sozialversicherungsbeiträge deutlich unter der vielfach als geradezu magisch angesehenen Obergrenze von $40 \%$, die auch im Koalitionsvertrag von CDU/CSU und SPD vom 11.11.2005 als zu unterschreiten postuliert wurde. ${ }^{8}$

\subsection{ABBAU DER "FEHLFINANZIERUNG"}

Beitragsbelastung wie auch Lohnnebenkosten können reduziert werden, wenn „Fehlfinanzierung" beseitigt wird. Angesetzt werden kann dabei an der Höhe der Ausgaben insgesamt, was den Finanzbedarf und, bei Abbau von Umverteilungsaufgaben in der Sozialversicherung, auch die "Fehlfinanzierung" reduziert, und/oder an der Struktur der Einnahmen bei unverändertem Finanzbedarf.

Auf den letztgenannten Fall - also eine Änderung der Einnahmestruktur - beschränke ich mich hier und gehe dabei nur auf einen der Ansatzpunkte ein, und zwar

\footnotetext{
7 Für die soziale Pflegeversicherung machen die familienpolitischen Leistungen etwa 0,25 Beitragspunkte aus.

8 Dass diese Marke zum Jahresanfang 2009 verfehlt wurde, führt die Bundesvereinigung der Deutschen Arbeitgeberverbände allein auf ausgabenerhöhende politische Entscheidungen zurück - die negativen Wirkungen auf die Beitragsbasis finden keine Erwähnung (Bundesvereinigung 2009a, S. 26). Eine Senkung des Gesamtsozialversicherungsbeitragssatzes unter $40 \%$ bleibe "richtig und unverzichtbar. Der Senkung der lohnbezogenen Sozialabgaben kommt [...] entscheidende Bedeutung für die Schaffung neuer Beschäftigung zu“ (ebd.); kritisch zu dieser Aussage: Schmähl (2009b). Zwar wird zum 1.7.2009 die Summe der Beitragssätze durch die vorgezogene Erhöhung der Bundeszahlungen an die GKV 39,6 \% betragen, was aus Sicht der BDA aber nur die "zweitbeste" Lösung ist: Ausgabensenkungen wären vorzuziehen (Bundesvereinigung 2009b).
} 
die Substitution von lohn- bzw. lohnersatzbezogenen Sozialbeiträgen durch Finanzierungsmittel aus Haushalten öffentlicher Gebietskörperschaften. Sie kann auf zwei Wegen erfolgen:

(a) durch eine organisatorisch-institutionelle Ausgliederung von Ausgaben, die bisher in der Sozialversicherung abgewickelt wurden, und deren Verlagerung in Haushalte öffentlicher Gebietskörperschaften, wo sie dann in der Regel aus Steuereinnahmen finanziert werden;

(b) durch eine finanzierungstechnisch-fiskalische Veränderung - eine Umfinanzierung -, indem die entsprechenden Ausgaben zwar weiter in der Sozialversicherung abgewickelt werden, jedoch nicht mehr durch Beiträge, sondern aus Steuern finanziert werden. Sofern davon abgesehen wird, durch Verminderung anderer Ausgaben einen entsprechenden Finanzierungsspielraum zu schaffen, ist zu klären, durch welche Steuern dies erfolgen soll.

Welche Auswirkungen könnte solch ein (aufkommensneutraler) Ersatz von Beitrags- durch Steuerfinanzierung für Beschäftigung und Einkommensverteilung, aber gegebenenfalls auch für die Konzeption des betreffenden Sozialversicherungszweiges haben? Bei der Beantwortung dieser Frage ist neben der Senkung der Sozialbeiträge stets auch die jeweilige Gegenfinanzierung durch Steuern zu berücksichtigen.

Die für Deutschland vorliegenden Simulationsberechnungen, auf die überblicksartig in Schmähl (2007a) eingegangen wird, zeigen, dass eine aufkommensneutrale Umfinanzierung nur vergleichsweise geringe beschäftigungserhöhende Effekte haben dürfte. Bei der beschäftigungspolitischen Begründung für den Abbau „überhöhter" Beiträge wird zumeist allein deren Reduzierung, aber nicht die Gegenfinanzierung thematisiert.

Über die Auswirkungen einer Minderung oder Beseitigung der Fehlfinanzierung durch Umfinanzierung auf die personelle Einkommensverteilung liegen bislang keine umfassenden, empirisch fundierten Analysen vor. Sie erfordern auch sehr komplexe Ansätze. So wären hierbei verschiedene Personengruppen zu unterscheiden, wie sozialversicherungspflichtig Beschäftigte mit einem Lohn bis zur oder über der Beitragsbemessungsgrenze, nicht sozialversicherungspflichtig Erwerbstätige, wie Grup- pen von Arbeitnehmern, z. B. Beamte, und verschiedene Gruppen von Selbstständigen sowie Nichterwerbstätige, wie Rentner und Pensionäre, jeweils vor allem hinsichtlich der Höhe und Zusammensetzung ihres Einkommens sowie ihrer Verbrauchsstruktur. Ferner kommt es darauf an, welche Steuern an die Stelle eines Teils der Arbeitnehmer- und Arbeitgeberbeiträge treten.

Hinsichtlich der Konzeption des jeweiligen Sicherungssystems erfolgt bei Beseitigung der Fehlfinanzierung durch Umfinanzierung eine Stärkung des Versicherungsgedankens insbesondere dort, wo die Höhe des Beitrags Folgen für die Höhe der Gegenleistung hat, also vor allem in der GRV, aber auch zum Teil in der Arbeitslosenversicherung. Hierdurch erhält der Beitrag stärker den Charakter eines Preises für den Erwerb von Ansprüchen, was für die Akzeptanz des Systems bzw. die Bereitschaft, zur Finanzierung beizutragen, von Bedeutung ist. Insgesamt würden zudem die staatlich initiierten Verteilungsvorgänge transparenter, und bei der Offenlegung von Kriterien für die Bemessung der Steuerzuschüsse an die Sozialversicherungsträger würden diese auch überprüfbar.

In verschiedenen Ländern - wie auch auf der EU-Ebene - wird in neuerer Zeit eine Stärkung des „Äquivalenzgedankens“ im Bereich sozialer Sicherung, und zwar gerade auch in der Sozialversicherung, befürwortet (Schmähl 2007b). Man könnte sich - insbesondere in der GRV - als Leitlinie für deren weitere Gestaltung sogar vorstellen, dass Ansprüche in der Regel nur auf der Basis von Beiträgen erworben werden. Wie im Falle der Kindererziehung können diese Beiträge auch von öffentlichen $\mathrm{Ge}-$ bietskörperschaften gezahlt werden. Ähnliches läge z. B. nahe, wenn Zeiten der Ausbildung rentensteigernd berücksichtigt werden sollen. Auch Beiträge anderer Sozialversicherungsträger - wie in Deutschland solche der Bundesagentur für Arbeit an die GRV zugunsten von Rentenansprüchen Arbeitsloser - sind ein weiteres Beispiel für eine derartige Strategie.

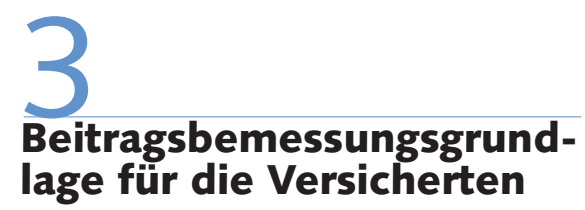

In Deutschland, aber auch in verschiedenen anderen Ländern, wird immer wieder über Änderungen der Bemessungsgrundla- ge für Sozialabgaben diskutiert. In Deutschland werden Sozialversicherungsbeiträge im Falle versicherungspflichtig Beschäftigter vom versicherungspflichtigen Bruttoarbeitsentgelt erhoben, jedoch nur bis zur jeweiligen Beitragsbemessungsgrenze. Darüber hinaus erfolgen unter bestimmten Bedingungen Beitragszahlungen auf der Basis von Lohnersatzleistungen, wie beispielsweise von Renten aus der GRV oder von Betriebsrenten für Beiträge zur Krankenversicherung. ${ }^{9}$

\subsection{LOHNBEZOGENE BEMESSUNGSGRUNDLAGE}

Mit Blick auf diese lohnbezogene Bemessungsgrundlage gibt es Änderungsvorschläge, die sich zum einen auf den unteren Lohnbereich beziehen, indem insbesondere eine für Geringverdiener („geringfügig Beschäftigte") bestehende Freigrenze (Geringfügigkeitsgrenze, auch „Gleitzone“) erhöht oder gesenkt werden soll und/oder durch Einführung eines Freibetrages in den Beitragstarif eine Minderung der Zahllast vor allem im unteren Lohnbereich angestrebt wird (beispielsweise Kaltenborn et al. 2003).

Sollen z. B. Geringverdiener bei ihrem Arbeitnehmerbeitrag oder deren Arbeitgeber insbesondere aus beschäftigungspolitischen Gründen hinsichtlich des Arbeitgeberbeitrags finanziell entlastet werden, so stellt sich die Frage, ob dies Auswirkungen auf die Höhe der Ansprüche der Versicherten hat (was nur bei Geldleistungen relevant ist), und wenn nicht, wie (und von wem) die damit verbundenen Beitragsmindereinnahmen kompensiert werden sollen. Die gleiche Frage stellt sich bei der Einführung von Freibeträgen.

Zum anderen wird mit Blick auf den oberen Lohnbereich immer wieder eine Anhebung oder gar Aufhebung der Beitragsbemessungsgrenze gefordert. Erfolgen in einem Versicherungszweig vor allem Sachleistungen (wie in der GKV), so führt eine höhere Beitragsbemessungsgrenze bei unverändertem Beitragssatz zu Mehreinnahmen bzw. es kann, da die Bemessungsgrundlage ausgedehnt wird, der Beitragssatz gesenkt werden. Anders verhält es sich, wenn wie in der GRV die Beitragszahlun-

9 Auf Beitragszahlungen des Staates an die Rentenversicherung zugunsten von Kindererziehenden wurde bereits hingewiesen. Rentenversicherungsbeiträge zahlen auch die Pflegekassen zugunsten Pflegeleistungen erbringender Personen. 
gen Auswirkungen auf die Höhe der Ansprüche haben: Dann stehen den erhöhten Einnahmen infolge der höheren Beitragsbemessungsgrenze später auch höhere Ausgaben gegenüber, sofern dieser Effekt nicht dadurch reduziert wird, dass die Beiträge vom Lohn oberhalb der bisherigen Beitragsbemessungsgrenze nur zu vergleichsweise geringeren Ansprüchen führen. Dies berührt aber unmittelbar die Konzeption des Systems, da hierdurch die interpersonelle Umverteilungswirkung erhöht wird und das Rentenversicherungssystem mehr in Richtung eines Steuertransfersystems rückt.

\section{2 ÜBER DEN LOHN HINAUSREICHENDE, BREITERE BEMESSUNGSGRUNDLAGE}

Diskutiert wird auch eine Ausweitung der Bemessungsgrundlage für Sozialabgaben auf weitere Einkunftsarten. Solch eine breitere Bemessungsgrundlage für Versicherte wird oftmals zusammen mit einer Ausweitung des zur Abgabe heranzuziehenden Personenkreises, namentlich der Selbstständigen und Beamten, diskutiert. Eine Bemessungsgrundlage, die alle Einkunftsarten des Versicherten bzw. des Privathaushalts umfasst, wäre in der Wirkung faktisch analog einer - allerdings proportionalen - Einkommensteuer (sofern keine Beitragsbemessungsgrenze oder Freibeträge existieren), begründet mit einer entsprechenden finanziellen Leistungsfähigkeit der Abgabepflichtigen, die über Löhne oder Lohnersatzleistungen hinausreicht. Dies könnte sich aber nur auf die Abgabe der Versicherten, nicht auch auf deren Arbeitgeber beziehen. Was mit den Arbeitgeberzahlungen passieren soll, ist eine offene Frage. Zumindest würde in der Regel keine paritätische Finanzierung erfolgen, was allerdings bereits jetzt durch Zusatzbeiträge der Fall ist.

Die Wirkungen einer solch breiteren einkommensbezogenen Bemessungsgrundlage wären in den verschiedenen Versicherungszweigen unterschiedlich. Da die Ausgaben für Sachleistungen in der GKV weitgehend unverändert bleiben würden, sofern nicht auch der Personenkreis erweitert wird, könnte der Abgabensatz ceteris paribus reduziert werden. Insgesamt würde dies zu Änderungen der personellen Belastungsverteilung zwischen den Privathaushalten führen. Der Charakter des Krankenversicherungssystems würde jedoch im Prinzip nicht verändert. ${ }^{10}$
Anders ist die Situation in der GRV, sofern hier weiterhin eine gewisse Äquivalenz zwischen Höhe der Abgabe und Höhe des Anspruchs besteht. ${ }^{11}$

Bislang stellt die Rente in Deutschland Lohnersatz dar. Wenn nun auch andere Einkunftsarten, wie Vermögenseinkünfte, in die Bemessungsgrundlage einbezogen werden, diese Einkünfte aber im Alter im Zweifel nicht entfallen und insofern auch nicht „ersetzt" werden müssen, dann ist das Gesamteinkommen systematisch auch nicht die geeignete Bemessungsgrundlage.

Wird jedoch mit einer solchen Ausweitung der Bemessungsgrundlage zugleich eine Abkehr von der proportionalen Beziehung zwischen Beitragshöhe und Höhe des Rentenanspruchs angestrebt, ähnlich wie etwa in der stark interpersonell umverteilenden Alters- und Hinterlassenenversicherung (AHV) der Schweiz, dann wäre damit eine Änderung der Systemkonzeption verbunden: Maßgebend wären die interpersonelle Umverteilung und die fiskalische Leistungsfähigkeit des Einzelnen, nicht aber eine enge Leistungs-GegenleistungsBeziehung. ${ }^{12}$

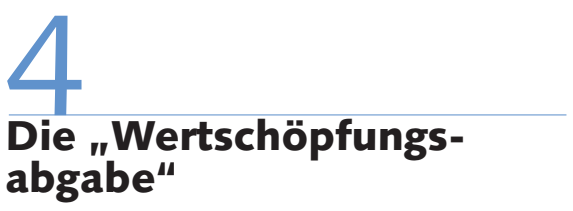

Die Diskussion über die Ausweitung der Bemessungsgrundlage für Arbeitgeberzahlungen insbesondere als Wertschöpfungsabgabe besitzt in mehreren „Sozialversicherungsländern“" wie Belgien, Frankreich, Österreich und Deutschland bereits eine längere Tradition. Eine solche Abgabe wurde entweder als Ersatz der lohnbezogenen Bemessungsgrundlage gefordert (also eine Umbasierung) oder als zusätzliche ergänzende Abgabe zur Finanzierung von (steigenden) Sozialleistungen vorgeschlagen.

Mit einer Umbasierung - sie wurde in Deutschland primär mit Blick auf die Rentenversicherung diskutiert - wurden zunächst strukturelle Effekte angestrebt, so eine wettbewerbspolitisch begründete Kostenminderung für lohnintensiv produzierende kleinere und mittlere Unternehmen durch Entlastung des Faktors Arbeit und Mehrbelastung kapitalintensiver Produktionen sowie, umweltpolitisch motiviert, auch solcher Unternehmen, die die natürliche Umwelt (Boden, Wasser, Luft) stark nutzen. Unternehmen sollten gemäß ihrer fiskalischen Leistungsfähigkeit und nicht nur entsprechend der versicherungspflichtigen Lohnsumme zur Finanzierung von Sozialausgaben herangezogen werden. Später rückte der Finanzierungsaspekt einer Wertschöpfungsabgabe immer stärker in den Vordergrund: Eine breitere Finanzierungsbasis sollte angesichts einer in $\mathrm{Zu}$ kunft erwarteten weiter sinkenden Lohnquote zu einer ergiebigeren Finanzierungsquelle werden. Der Finanzierungsaspekt stand auch im Vordergrund beim Vorschlag, einen künftig steigenden Finanzbedarf nicht oder nicht allein durch höhere lohnbezogene Beitragseinnahmen, sondern auch durch eine ergänzende Wertschöpfungsabgabe zu decken (Schmähl 2008a, S. 492ff.; Schmähl et al. 1984).

Im Unterschied zu den Argumenten der Befürworter einer Umbasierung erbrachten die für Deutschland insbesondere in den 1980er Jahren durchgeführten Analysen allerdings recht ernüchternde Ergebnisse. So stellen die mit einer Umbasierung verbundenen Änderungen der Kostenstruktur der Unternehmen nur die erste Stufe eines komplexen Wirkungsgeflechts dar: Es reicht nicht, allein die durchschnittlichen Belastungsänderungen für ganze Wirtschaftszweige zu berücksichtigen, denn auch innerhalb eines Wirtschaftszweiges kann es zu beträchtlichen Veränderungen zwischen Unternehmen kommen, wie anhand einer Analyse für den deutschen Einzelhandel gezeigt wurde (Rüdiger 1992). Auch die intraindustrielle Produktionsverflechtung ist zu beachten und vor allem, wie Unternehmen auf Veränderungen der Kostenstruktur reagieren. Einfache Aussagen sind kaum möglich.

10 Eine völlige Abkoppelung vom Arbeitsverdienst würde im Falle der Einführung von einheitlich hohen Kopfbeiträgen erfolgen. Sie wären unabhängig von der Einkommensquelle aus dem jeweiligen Haushaltseinkommen aufzubringen, würden stark regressiv wirken und müssten im Zweifel in bestimmten Einkommensbereichen durch Steuermittel subventioniert werden. Dies wurde in jüngster Zeit in Deutschland für die Krankenversicherung diskutiert.

11 Durch die Veränderung der Bemessungsgrundlage würden sich allerdings die individuellen relativen Ansprüche verändern, wenn, wie in Deutschland, diese auf der Relation individueller Einkommen zum Durchschnittseinkommen aller basieren. Darauf wird hier nicht eingegangen.

12 Ein solcher Systemwechsel von der GRV zu einem staatlichen Umverteilungssystem mit niedrigem Leistungsniveau sollte allerdings nicht isoliert gesehen werden, sondern Folgewirkungen, wie z. B. ein mögliches Obligatorium für Betriebsrenten - siehe wiederum die Schweiz -, sind einzubeziehen. 
Für Deutschland durchgeführte Untersuchungen zeigen (Meinhardt/Zwiener 1997, S. 352ff.; Walwei/Zika 2005, S. 77ff.), dass zwar in einer Unterbeschäftigungssituation von einer Umbasierung ein positiver Beschäftigungseffekt ausgehen kann, dieser allerdings recht gering ist. Für das Wirtschaftswachstum können dann negative Konsequenzen eintreten, wenn durch eine stärkere Belastung des Faktors Kapital die Investitionstätigkeit negativ berührt wird, was wiederum entsprechende Konsequenzen für die fiskalische Ergiebigkeit der Abgabe hätte. In der deutschen Diskussion spielt die Wertschöpfungsabgabe als Finanzierungsquelle für Sozialleistungen allerdings derzeit kaum eine Rolle.

\section{5 \\ Mehr Steuerfinanzierung in der Sozialversicherung?}

Ein höheres Maß an Steuerfinanzierung in der Sozialversicherung kann bei klar definierter Abgrenzung derjenigen Aufgaben, die als allgemeine Staatsaufgaben anzusehen sind, verteilungs- und beschäftigungspolitisch positiv wirken und $\mathrm{zu}$ mehr Transparenz der Verteilungsvorgänge wie auch zu einer stärkeren Orientierung am Versicherungsgedanken in der Sozialversicherung beitragen und damit zugleich deren Akzeptanz erhöhen. ${ }^{13}$

\subsection{HÖHERER STEUERANTEIL IN DER GRV}

Steuermittel sind derzeit im Bereich der Alterssicherung nicht nur wegen der Bundeszahlungen an die GRV von Bedeutung. Sie spielen auch im Rahmen der betrieblichen Alterssicherung traditioneller Art und im Zuge der Entgeltumwandlung sowie für die sonstige geförderte private Vorsorge eine Rolle. Entgeltumwandlung und Förderung privater Vorsorge gehören mit zu den zentralen Maßnahmen des grundlegenden Paradigmenwechsels in der deutschen Alterssicherungspolitik, der ab 2001 politisch umgesetzt wurde. Die damit verbundenen tief greifenden Folgen für die Versicherten (Schmähl 2008b) wurden allerdings zunächst „nachhaltig“ ignoriert, geraten jedoch inzwischen immer mehr ins Blickfeld der Menschen und werden nun auch von den Medien aufgegriffen.
Diese beginnen, Politiker und Politikberater zu Reaktionen herauszufordern.

Die dem politischen Handeln zugrundeliegenden Ziele (auch nach dem Regierungswechsel von der Rot-Grünen zur Großen Koalition) und die damit verbundenen Interessen wurden an anderer Stelle dargelegt (Schmähl 2008b; Viebrok et al. 2004). Im Vordergrund stehen die Dominanz des Beitragssatzzieles, die Reduzierung des Leistungsniveaus der GRV um etwa $25 \%$ durch Einfügung verschiedener "Faktoren" in die inzwischen völlig intransparente Rentenformel, der partielle Ersatz der GRV u. a. durch die beitragsfreie Entgeltumwandlung und sonstige marktabhängige private Alterssicherungsprodukte. Die Folgen sind beträchtlich:

Erstens entsteht für lange Zeit eine höhere finanzielle Belastung der Privathaushalte in der Erwerbsphase durch Beitragszahlungen zur GRV und zu privaten bzw. über Betriebe abgewickelten Vorsorgemaßnahmen, als sie bei vergleichbarem Absicherungsniveau allein durch die GRV erforderlich wäre. Zweitens erfolgt eine Verlagerung von Risiken von öffentlichen Haushalten und Arbeitgebern hin zu Privathaushalten.

Drittens wächst die Gefahr deutlich, dass angesichts des erheblich reduzierten Leistungsniveaus in der gesetzlichen Rentenversicherung selbst nach langem Erwerbsleben die in der GRV erworbene Rente vielfach unter einer armutsvermeidenden Grundsicherung (Sozialhilfe) liegen wird. Hinzu kommt die durch Arbeitslosigkeit und zunehmende Diskontinuität von Versicherungsverläufen verminderte Möglichkeit, ausreichende Rentenansprüche zu erwerben. Bei den Haushalten, deren Alterseinkommen allein oder weit überwiegend aus Renten bestehen, werden diese Folgen am stärksten zu Buche schlagen, so auch in besonderem Maße in Ostdeutschland.

Viertens besteht die wachsende Gefahr wieder steigender Altersarmut, welche in der Vergangenheit durch die GRV weitgehend zurückgedrängt wurde. Dieses Risiko gilt besonders in Zeiten der Arbeitslosigkeit, in denen keine Betriebsrentenansprüche erworben werden und kaum private Vorsorge erfolgen kann. Stark betroffen sind auch Erwerbsunfähigkeitsrentner, da sie einerseits von der Reduktion des Leistungsniveaus in der GRV erfasst werden, andererseits für sie aufgrund der vorzeitigen Beendigung der Erwerbsarbeit kein potenzielles „Auffangnetz“ durch be- triebliche oder geförderte private Vorsorge mehr existiert.

Schließlich drohen eine steigende Einkommensungleichheit im Alter sowie eine Transformation der GRV durch den bereits begonnenen Ausstieg aus der lohnbezogenen GRV mit ihrem Lohnersatzcharakter zu einem gesetzlichen System, das primär dem Ziel dient, (das Ende des 19. Jahrhunderts Anlass für die Einführung der Sozialversicherung war), nur einen Beitrag zur Vermeidung von Einkommensarmut im Alter zu leisten.

Sollen diese Folgen des in Deutschland zu Beginn des Jahrtausends beschlossenen „Paradigmenwechsels“ in der Alterssicherung noch vermieden werden, dann wäre insbesondere erforderlich, dass in der GRV langjährig Versicherte einen Rentenanspruch aus ihren Beitragszahlungen realisieren können, der spürbar über dem Grundsicherungs- bzw. Sozialhilfeniveau liegt. Dies bedeutet, dass der programmierte Rückbau des Leistungsniveaus schnellstmöglich gestoppt werden müsste. Dies ist ohne Verletzung des politisch postulierten Beitragssatzzieles insbesondere dann möglich, wenn in der GRV eine aufgabenadäquate Finanzierung erfolgen würde, und zwar vor allem für die Ausgaben der Hinterbliebenenversorgung. Dies sei kurz konzeptionell begründet:

Durch die 1986 in Kraft getretene gesetzliche Neuregelung werden Hinterbliebenenrenten nur noch ausgezahlt, wenn eigene (mit einem bestimmten Prozentsatz) anrechenbare Einkünfte des überlebenden Ehegatten unter einem bestimmten (Frei-)Betrag bleiben. Nach den Entscheidungen zur „Rentenreform 2001“ werden in Zukunft alle Arten von Einkünften (bis auf die geförderte Privatrente) auf Hinterbliebenenrenten angerechnet. Es wird also geprüft, ob der/die Hinterbliebene dieser Leistung überhaupt „,bedarf“. Für bedarfsgeprüfte Leistungen (wie z. B. Wohngeld oder Sozialhilfe) sind aber Sozialbeiträge das falsche Finanzierungsinstrument. Die Ausgaben für die Hinterbliebenenversorgung betragen rund ein Fünftel der Rentenausgaben. D. h., der Beitragssatz zur gesetzlichen Rentenversicherung wür-

\footnotetext{
13 Die nun begonnene Steuerfinanzierung in der GKV ist im Prinzip ein richtiger Weg. Wenn insbesondere familienpolitische Leistungen finanziert werden sollen, so stellt sich hier wie auch generell die Frage, ob diese allein für Familien von Sozialversicherungspflichtigen wirksam werden sollen.
} 
de bei aufgabenadäquater Finanzierung dieser Leistungen um gut 3,5 Beitragspunkte niedriger liegen (Schmähl 2007a). Würde realistischerweise in Stufen entsprechend umfinanziert werden, so könnte für einen langen Zeitraum das gegenwärtige Leistungsniveau der GRV ohne Steigerung des Beitragssatzes erhalten bleiben und die beschriebenen negativen Folgen der derzeitigen Strategie könnten vermieden werden. ${ }^{14}$

Diese Umfinanzierung der Hinterbliebenenrenten wäre folglich ein maßgebendes Element, um die GRV mit Lohnersatzcharakter zu erhalten und zumindest für langjährig Versicherte ein Rentenniveau zu realisieren, welches spürbar das Niveau einer armutsvermeidenden bedürftigkeitsgeprüften Grundsicherung übersteigt. Damit würden der derzeitige Demontageprozess der GRV gestoppt, die Beziehung zwischen Vorsorgebeitrag und späterer Rente gestärkt (Beitragszahlung lohnt sich) und die Grundlage für ein Alterseinkommen geschaffen, damit man im Alter ,in Würde“ leben kann, wie dies auch der neue amerikanische Präsident in seiner Antrittsrede als eine Aufgabe des Staates hervorhob (Obama 2009). Damit würde auch die Vorsorgebereitschaft nicht nur in der gesetzlichen, sondern auch der privaten Alterssicherung gestärkt und insgesamt würden sogar im Vergleich zur jetzt politisch implementierten Strategie die Vorsorgeaufwendungen für die privaten Haushalte reduziert.

Ein weiteres Element einer Strategie, die die lohnbezogene Rentenversicherung erhalten und zugleich einen neuerlichen
Anstieg von Altersarmut durch vermehrte Steuerfinanzierung wirksam verhindern will, wäre eine deutliche Erhöhung der nur noch minimalen Rentenbeiträge der Bundesagentur für Arbeit für Bezieher von Arbeitslosengeld II, ${ }^{15}$ die im Interesse der Entlastung des Bundes reduziert wurden. Die Erhöhung des Steueranteils in der Sozialversicherung gehört also auf die politische Tagesordnung, nicht nur in der GKV, sondern auch in der GRV.

Die Selbstständigen, die bislang nicht von einem obligatorischen Alterssicherungssystem erfasst sind, in die GRV einzubeziehen (also deren „Pflichtversicherung "), ${ }^{16}$ würde dazu beitragen, die Gefahr von Altersarmut in dieser Gruppe zu verringern, indem längere sonst gegebenenfalls nicht oder nur unzureichend abgesicherte Lebensphasen vermieden werden (Fachinger et al. 2004; Ehler/Frommert 2009).

Von zentraler Bedeutung ist aber, dass in der GRV politisch möglichst bald umgesteuert wird, indem ein ausreichendes Leistungsniveau wieder maßgebendes Ziel wird und die Lohnbezogenheit der Rente bei ihrer Erstberechnung und während der Laufzeit wiederhergestellt wird. Lange darf man allerdings mit einer politischen Kurskorrektur nicht warten. Denn ist erst einmal das Leistungsniveau in der GRV deutlich gesunken, so wird es kaum wieder anzuheben sein. ${ }^{17}$ Dem Versuch, sich auf die Grundkonzeption des Rentenversicherungssystems rückzubesinnen, wie sie 1957 geschaffen wurde, werden sich allerdings einflussreiche Interessengruppen widersetzen, die zu den Verfechtern und Gewinnern des politisch durchgesetzten und angeblich „alternativlosen Paradigmenwechsels“ der letzten Jahre gehören. ${ }^{18}$ Und ob die Politiker, die den „Paradigmenwechsel“ durchsetzten und/oder begrüßten, den Mut zu einer solchen Kurskorrektur haben, erscheint derzeit (noch) zweifelhaft. Doch je deutlicher die negativen Folgen des politischen Handelns in der Öffentlichkeit thematisiert werden, umso eher steigen die Chancen für einen Wandel.

14 Die Rentenformel sollte zudem einfach und verständlich werden, indem sie ausschließlich die Lohnentwicklung und Beiträge zur GRV enthält.

15 Siehe z. B. Wübbeke (2007, S. 103), die konstatiert, es sei absehbar, "dass für die heute 40- bis 50-jährigen Bezieher von ALG II das Risiko der Altersarmut generell steigt ".

16 Demgegenüber würde eine "Versicherungspflicht" die auch die Möglichkeit der Absicherung nur durch private Vorsorge zulässt, grundlegende Fragen auch im Hinblick auf die Legitimierbarkeit der Pflichtversicherung für unselbstständig Beschäftigte aufwerfen.

17 Die für betriebliche Alterssicherung und private Vorsorge eingesetzten öffentlichen Fördermittel sollten auf ihre Zielgenauigkeit überprüft werden, denn die Annahme ist plausibel, dass es hier in beträchtlichem Umfang zu Mitnahmeeffekten kommt und diese in besonderem Maße Haushalte mit höherem Einkommen begünstigen. Betriebliche Alterssicherung und private Vorsorge sind zweifellos wichtige Elemente des Alterssicherungssystems. Sie könnten und sollten allerdings wieder ihren Charakter als ergänzende und nicht, wie jetzt, ersetzende Formen der Vorsorge zurückerlangen.

18 So warnten u. a. der Sachverständigenrat zur Begutachtung der gesamtwirtschaftlichen Entwicklung und sein seinerzeitiger Vorsitzender, der an maßgebend beratender Stelle den Systemumbau propagierte, davor, an den Entscheidungen, die den „Paradigmenwechsel“ nach der Jahrtausendwende ausmachen, zu rütteln (Sachverständigenrat 2008, S. 385). 


\section{LITERATUR}

Bundesvereinigung der Deutschen Arbeitgeberverbände (2009a):

Geschäftsbericht 2008, Berlin

Bundesvereinigung der Deutschen Arbeitgeberverbände (2009b): Bei-

tragssatzsumme sinkt zur Jahresmitte wieder unter 40 Prozent, in: Soziale

Selbstverwaltung März 2009, S. 22

Deutsche Bundesbank (2008): Entwicklung der Steuereinnahmen in

Deutschland und aktuelle steuerpolitische Fragen, in: Monatsbericht

Oktober 2008, S. 35-59

Ehler, J./Frommert, D. (2009): Für eine Pflichtversicherung bei Selbststän-

digkeit ohne obligatorische Alterssicherung, in: Deutsche Rentenversiche-

rung 1, S. 36-57

Fachinger, U./Oelschläger, A./Schmähl, W. (2004): Alterssicherung von

Selbständigen - Bestandsaufnahme und Reformoptionen, Münster

Kaltenborn, B./Koch, S./Kress, U./Walwei, U./Zika, G. (2003): Arbeits-

markteffekte eines Freibetrags bei den Sozialabgaben, München und

Mering

Meinhardt, V./Zwiener, R. (1997): Steuerfinanzierung von versicherungsfremden Leistungen in der Sozialversicherung, in: Vierteljahreshefte zur Wirtschaftsforschung, S.352-361

Obama, B. (2009): Eine neue Ära der Verantwortung, in: Frankfurter Allgemeine Zeitung vom 22.1., S. 7

Rüdiger, D. (1992): Von bruttolohnbezogenen Arbeitgeber-Beiträgen zu wertschöpfungsbezogenen Arbeitgeber-Abgaben, Frankfurt/M. und Berlin

Ruland, F. (2009): Der Einzug von Sozialversicherungsbeiträgen in Tschechien - Zur Problematik des von der Weltbank propagierten Modells einer integrierten Einnahmenverwaltung, in: Zeitschrift für europäisches Sozialund Arbeitsrecht (ZESAR) 1, S. 14-20

Sachverständigenrat zur Begutachtung der gesamtwirtschaftlichen Entwicklung (2008): Die Finanzkrise meistern - Wachstumskräfte stärken. Jahresgutachten 2008/09, Wiesbaden

Schmähl, W. (2007a): Aufgabenadäquate Finanzierung der Sozialversicherung durch Beiträge und Steuern - Begründungen und Wirkungen eines Abbaus der "Fehlfinanzierung“ in Deutschland, in: Blanke, H.-J. (Hrsg.): Die Reform des Sozialstaats zwischen Freiheitlichkeit und Solidarität, Tübingen, S. 57-85
Schmähl, W. (2007b): Zur Entwicklung von Alterssicherungssystemen in Europa: Strukturen, Einflußfaktoren, Entwicklungstendenzen und Herausforderungen, in: Gesellschaft für Versicherungswissenschaft und -gestaltung (Hrsg.): Alterssicherung in Europa, Bonn, S. 11-42

Schmähl, W. (2008a): Sicherung bei Alter, Invalidität und für Hinterbliebene, in: Bundesministerium für Arbeit und Soziales und Bundesarchiv (Hrsg.): Geschichte der Sozialpolitik in Deutschland seit 1945. Band 6: 1974-1982 Bundesrepublik Deutschland - Neue Herausforderungen, wachsende Unsicherheit. Bandherausgeber Martin H. Geyer, BadenBaden, S. 393-514

Schmähl, W. (2008b): Die Gefahr steigender Altersarmut in Deutschland - Gründe und Vorschläge zur Armutsvermeidung, in: Richter, A./Bunzendahl, I./Altgeld, T. (Hrsg.): Dünne Rente-Dicke Probleme. Alter, Armut und Gesundheit - Neue Herausforderungen für Armutsprävention und Gesundheitsförderung, Frankfurt/M., S. 37-58

Schmähl, W. (2009a): Soziale Sicherung: Ökonomische Analysen, Wiesbaden

Schmähl, W. (2009b): Lohnnebenkosten, in: Gillen, G./Rossum, W. van (Hrsg.): Schwarzbuch Deutschland - Das Handbuch der vermissten Informationen, Reinbek, S. 406-415 und S. 623-625

Schmähl, W./Henke, K.-D./Schellhaaß, H. M. (1984): Änderung der Beitragsfinanzierung in der gesetzlichen Rentenversicherung? - Ökonomische Wirkungen des Maschinenbeitrags, Baden-Baden Schmähl, W./Oelschläger, A. (2007): Abgabenfreie Entgeltumwandlung aus sozial- und verteilungspolitischer Perspektive (Beiträge zur Sozial- und Verteilungspolitik, Bd. 5), Berlin

Viebrok, H./Himmelreicher, R. K./Schmähl, W. (2004): Private Vorsorge statt gesetzlicher Rente: Wer gewinnt, wer verliert? (Beiträge zur Sozialund Verteilungspolitik, Bd. 3), Münster und Hamburg Walwei, U./Zika. G. (2005): Arbeitsmarktwirkungen einer Senkung der Sozialabgaben, in: Sozialer Fortschritt 4, S. 77-90

Wübbeke, C. (2007): Ende gut, alles gut? Wenn Hilfeempfänger in Rente gehen, in: IABFORUM 1, S. 100-104 